DFTT 35/93

June 1993

\title{
Pion Compton Scattering in Perturbative QCD
}

\author{
Ezio Maina and Roberto Torasso \\ Dipartimento di Fisica Teorica, Università di Torino, Italy \\ and INFN, Sezione di Torino, Italy
}

\begin{abstract}
Pion Compton scattering is studied in perturbative QCD for real and space-like initial photons. Different methods for the convolution of the hard amplitude with the pion wave-functions, which have in the past led to conflicting results, are compared.
\end{abstract}

${ }^{1}$ Work supported in part by Ministero dell' Università e della Ricerca Scientifica. 


\section{Introduction}

The analysis of exclusive processes in QCD has vastly improved in the last few years. The rôle of Sudakov logarithms in elastic scattering has been investigated [1]. It has been shown [2] that, like form factors and $\gamma \gamma$ reactions, large angle photoproduction and Compton scattering are not affected in lowest order by Sudakov corrections and that, as a consequence, the magnitude and the phase of the scattering amplitudes for these processes can be reliably predicted by perturbative QCD (pQCD) at large momentum transfer. The applicability of pQCD to hadron form factor has been extended to lower energies using an improved version of the factorization formula [3]. The sum-rule approach has been applied to Compton scattering [4, 5].

Nonetheless, the number of reactions for which theoretical predictions exist is still rather small. The difficulties are best exemplified by the existence of three published calculations for proton Compton scattering, $\gamma p \rightarrow \gamma p$, in disagreement with each other 6, 7, 8].

In this paper we consider pion Compton scattering for real and space-like initial photons in pQCD. Pion Compton is the simplest reaction in which non trivial-phases are present and which is experimentally accessible. Its calculation is conceptually similar to the calculation of proton Compton scattering, but much simpler. It is, therefore, an ideal laboratory for studying the different methods [6, 7, 8] for the convolution of the hard amplitude.

Pion Compton has been considered before for real [5, 9] and time-like photons [9], in this paper we extend previous calculations to new kinematical domains and correct some errors that are present in [9].

There are different ways of measuring the $\pi \gamma$ cross section. In the first instance a beam of pions is scattered off of a nucleus. In the forward direction the one-photonexchange mechanism dominates [10] and $\pi \gamma$ scattering can be studied. Alternatively the interaction can be between an high-energy electron beam and a nucleus, with the extraction of quasi-real pions from nuclear matter.

\section{Calculation}

Computing the amplitude for an exclusive process involves two steps. First of all, an analytical expression for the large set of diagrams which is often required needs to be derived. This is usually far from trivial and in some cases can only be accomplished by some automatic procedure. Second, the result has to be multiplied by the wave functions of all external hadrons and then it has to be integrated over the momentum fractions of all external quarks. Apart from simple cases like lepton-hadron scattering and photon-photon annihilation, for particular values of the quark momenta, one or more of the particles propagating inside the various diagrams can go on their mass-shell. Mathematically this means that one-dimensional integrals of the form:

$$
\lim _{\epsilon \rightarrow 0} \int_{0}^{1} \frac{f(x)}{x-a+i \epsilon} d x=P \int_{0}^{1} \frac{f(x)}{x-a} d x-i \pi f(a)
$$

where $0<a<1$, have to be computed, and that the result has to be integrated over all remaining variables. 
Several methods have been proposed but unfortunately when applied to the reaction $\gamma p \rightarrow \gamma p$ they have led to conflicting results. Therefore we have preferred not to rely on only one method of integration. This also allows us to compare the different procedures and to study their relative merits.

We have considered a simpler version of the method used in [6], which we will call the subtraction method, the technique suggested in [7], which we will call the KN method and the procedure proposed in [8], which will be referred to as the $i \epsilon$ method.

In the first two cases some transformation is applied to the principal part integral in the right-hand side of (1) in order to avoid the large cancellations involved which lead to large numerical instabilities. The imaginary part is then computed by hand, a procedure that is rather tedious and that, if not automated, can be a source of errors which are almost impossible to detect.

The subtraction method is based on the following identity:

$$
\begin{aligned}
P \int_{0}^{1} \frac{f(x)}{x-a} d x & =\int_{0}^{1} \frac{f(x)-f(a)}{x-a} d x+f(a) P \int_{0}^{1} \frac{1}{x-a} d x \\
& =\int_{0}^{1} \frac{f(x)-f(a)}{x-a} d x+f(a) \log \frac{1-a}{a}
\end{aligned}
$$

Both resulting integrals are numerically stable.

The KN method relies on a change of variable to simplify the evaluation of the real part of eq. (1). One can write:

$$
P \int_{0}^{1} \frac{f(x)}{x-a} d x=\lim _{\epsilon \rightarrow 0}\left(J_{1}+J_{2}\right)
$$

where

$$
J_{1}=\int_{0}^{a-\epsilon} \frac{f(x)}{x-a} d x \quad J_{2}=\int_{a+\epsilon}^{1} \frac{f(x)}{x-a} d x
$$

Now the following change of variables is performed on $J_{1}$ :

$$
y_{1}=\frac{x}{a} \Rightarrow J_{1}=\int_{0}^{1-\epsilon / a} \frac{f\left(a y_{1}\right)}{y_{1}-1} d y_{1}
$$

and on $J_{2}$ :

$$
y_{2}=\frac{a(1-x)}{a^{2}+(1-2 a) x} \Rightarrow J_{2}=\int_{0}^{1-\epsilon / a} \frac{(1-a) f(g)}{d(1-y)} d y_{2}
$$

where

$$
g=\frac{a(1-a y)}{d} \quad d=a+(1-2 a) y
$$

The integration variables $y_{1}, y_{2}$ satisfy:

$$
\left.\frac{d x}{d y_{1}}\right|_{y_{1}=1}=-\left.\frac{d x}{d y_{2}}\right|_{y_{2}=1}
$$

therefore the upper limits in (5, 6) approach 1 uniformly. From (5, 6) one can derive an expression for the principal part of the integral in which the limit $\epsilon \rightarrow 0$ can be safely taken: 


$$
P \int_{0}^{1} \frac{f(x)}{x-a} d x=\int_{0}^{1}\left\{\frac{f(a y)}{y-1}+\frac{(1-a) f(g)}{d(1-y)}\right\} d y
$$

The last integral is well behaved and can be directly integrated numerically.

In our experience the subtraction method and the KN method always give the same results and can be implemented with comparable effort.

The $i \epsilon$ method is the easiest one to implement automatically on the large set of diagrams required to describe exclusive scatterings at tree level. It has the additional advantage that the real and imaginary part of eq. (11) are generated with the same algorithm and that, in principle, the integrand once generated is not manipulated any further, hence no mistakes are stirred in. The method consists in evaluating numerically the real and imaginary part of the integrals

$$
\int_{0}^{1} \frac{f(x)}{x-a+i \epsilon} d x
$$

for a number of small but finite values of $\epsilon$, since for too small values of $\epsilon$ numerical instabilities take over, and then to extrapolate to $\epsilon=0$. In [8] Farrar and Zhang noted that for $5 . \times 10^{-4}<\epsilon<1 . \times 10^{-2}$ the results seemed to depend very little on $\epsilon$ and therefore assumed that the amplitudes obtained for $\epsilon=5 . \times 10^{-3}$ could be taken as a good approximation to the $\epsilon=0$ results. This procedure was later criticized in [7] since it failed to reproduce the correct answer in a number of cases in which an analytical evaluation was feasible.

Our findings, for the much simpler set of diagrams we have been studying, are in agreement with those in [7]. For $\epsilon=5 . \times 10^{-3}$ the difference between the amplitudes evaluated with the $i \epsilon$ method and with the two others can be as large as $4 \div 5 \%$ and $20 \div 30 \%$ for the real and imaginary part of the amplitude respectively. For $\epsilon=5 . \times 10^{-4}$, a value for which the integrals show no sign of numerical problems, the difference can still be of several percent. In most cases a much better agreement, compatible with the numerical errors, can be obtained fitting a number of results in the range $5 . \times 10^{-4}<\epsilon<5 . \times 10^{-3}$ with a quadratic polynomial in $\epsilon$ whose coefficients are determined by the least squares method, and assuming the value of the polynomial for $\epsilon=0$ as the true result. Some caution is however needed. We have found instances, for perfectly reasonable values of the scattering angle and of the photon virtuality, in which the result is disturbingly sensitive to the chosen range. In some cases, in order to reproduce the correct result, the fit has to be performed on a different range, for instance $1 . \times 10^{-4}<\epsilon<1 . \times 10^{-3}$. The difference with the result obtained through a fit in the range $5 . \times 10^{-4}<\epsilon<5 . \times 10^{-3}$ can be as large as $10 \%$. This obviously casts some doubts on the possibility of a completely automatic implementation of the method.

As already noted [11], the limit in which the mass $q^{2}=-Q^{2}<0$ of the virtual photon vanishes is particularly tricky. For non-vanishing photon mass a larger number of propagators can go on mass-shell and, after partial fractioning, one discovers that different terms require different ranges in $\epsilon$ to give an acceptable extrapolation to $\epsilon=0$. 


\section{Results}

We define the scattering kinematics for $e\left(k, h_{e}\right) \pi(p) \rightarrow e\left(k^{\prime}, h_{e}\right) \pi\left(p^{\prime}\right) \gamma\left(q^{\prime}, \lambda\right)$ as in [8]. The initial and final pion and the final photon have momenta:

$$
\begin{aligned}
p & =P(1,0,0,-1) \\
p^{\prime} & =Q^{\prime}(1,-\sin \theta, 0,-\cos \theta) \\
q^{\prime} & =Q^{\prime}(1, \sin \theta, 0, \cos \theta)
\end{aligned}
$$

while momenta of the initial and final electrons are

$$
\begin{aligned}
k & =K(1, \cos \phi \sin \alpha, \sin \phi \sin \alpha, \cos \alpha) \\
k^{\prime} & =K^{\prime}\left(1, \cos \phi \sin \alpha^{\prime}, \sin \phi \sin \alpha^{\prime}, \cos \alpha^{\prime}\right)
\end{aligned}
$$

The diagrams contributing to $e \pi$ scattering can be divided into two sets. The first set includes all diagrams in which the final photon is emitted from the quark lines and will be referred to as the pion Compton set. In this case for the momentum of the intermediate photon we have

$$
q=\left(\sqrt{P^{2}-Q^{2}}, 0,0, P\right)
$$

The second set includes all diagrams in which the final photon is emitted from the electron line.

Momentum conservation requires the following relationships among the parameters

$$
\begin{aligned}
K \sin \alpha & =K^{\prime} \sin \alpha^{\prime} \\
P & =K \cos \alpha-K^{\prime} \cos \alpha^{\prime} \\
P+K & =2 Q^{\prime}+K^{\prime} \\
Q^{2} & =4 Q^{\prime}\left(P-Q^{\prime}\right)
\end{aligned}
$$

For a (virtual) photon propagating along the positive $z$-axis, the polarization vectors are defined in the standard way

$$
\epsilon_{L, R}^{\mu}=\frac{1}{\sqrt{2}}(0,-1, \pm i, 0) \quad \epsilon_{ \pm}^{\mu}=\frac{1}{\sqrt{2}}\left(\epsilon_{0}^{\mu} \pm \epsilon_{3}^{\mu}\right)=\frac{1}{\sqrt{2}}(1,0,0, \pm 1)
$$

Writing the amplitude $A_{h_{e}}^{\lambda}$ for the pion Compton diagrams as:

$$
A_{h_{e}}^{\lambda}=M_{\nu} g^{\mu \nu} V_{\mu \rho} \epsilon^{\rho *} / q^{2}
$$

where $M_{\nu}$ describes the emission of a virtual photon from the lepton line and $T_{\mu}=$ $V_{\mu \rho} \epsilon^{\rho *}$ describes the scattering of this photon off the pion, and using the identity

$$
-g^{\mu \nu}=\epsilon_{R}^{\mu} \epsilon_{R}^{\nu *}+\epsilon_{L}^{\mu} \epsilon_{L}^{\nu *}-\epsilon_{+}^{\mu} \epsilon_{-}^{\nu *}-\epsilon_{-}^{\mu} \epsilon_{+}^{\nu *}
$$

one can write

$$
-q^{2} A=M_{L} T_{L}+M_{R} T_{R}-M_{-} T_{+}-M_{+} T_{-}
$$


Gauge invariance of the amplitude $T_{\mu}$ requires

$$
\begin{aligned}
q \cdot T & =\sqrt{P^{2}-Q^{2}} T^{0}-P T^{3}=\sqrt{P^{2}-Q^{2}} \epsilon_{0} \cdot T+P \epsilon_{3} \cdot T \\
& =\frac{1}{\sqrt{2}} \sqrt{P^{2}-Q^{2}}\left(T_{+}+T_{-}\right)+\frac{1}{\sqrt{2}} P\left(T_{+}-T_{-}\right)=0
\end{aligned}
$$

It is therefore sufficient to give the amplitudes $V_{i j}=V_{\mu \nu} \epsilon_{i}^{\mu} \epsilon_{j}^{\nu *}$ for incoming photons with polarization $\epsilon_{L}, \epsilon_{R}$ and $\epsilon_{+}$.

All the computations have been made in the formalism of [12]. Our results for the unintegrated amplitudes coincide with those in [9]. In all formulae we use the abbreviations $c=\cos \theta / 2, c_{\alpha}=\cos \alpha / 2, c_{\alpha^{\prime}}=\cos \alpha^{\prime} / 2, s=\sin \theta / 2, s_{\alpha}=\sin \alpha / 2$, $s_{\alpha^{\prime}}=\sin \alpha^{\prime} / 2$.

It is amusing to note that the amplitude for $\pi \gamma_{L} \rightarrow \pi \gamma_{R}$ with real photons has a very simple form

$$
V_{L R}=C_{0}\left(e_{1}-e_{2}\right)^{2}(x-y) s^{2} / c^{2}
$$

where $C_{0}$ is a constant, $x$ and $y$ are the momentum fractions of the initial and final quark and $e_{1}$ and $e_{2}$ are the charges of the two quark lines. This is in agreement with a general theorem on helicity amplitudes [13].

We present only the amplitudes for right-handed final photons, since all others are related by parity. The helicity amplitudes $A_{h_{e}}^{\lambda}$ for the pion Compton contribution to $e \pi \rightarrow e \pi \gamma$, with the definitions given in (15), are

$$
\begin{aligned}
& A_{-}^{R}=c_{\alpha^{\prime}} c_{\alpha} V_{+R}+s_{\alpha^{\prime}} s_{\alpha} V_{-R}-e^{i \phi} s_{\alpha^{\prime}} c_{\alpha} V_{R R}-e^{-i \phi} c_{\alpha^{\prime}} s_{\alpha} V_{L R} \\
& A_{+}^{R}=c_{\alpha^{\prime}} c_{\alpha} V_{+R}+s_{\alpha^{\prime}} s_{\alpha} V_{-R}-e^{i \phi} c_{\alpha^{\prime}} s_{\alpha} V_{R R}-e^{-i \phi} s_{\alpha^{\prime}} c_{\alpha} V_{L R}
\end{aligned}
$$

times the common factor

$$
c_{A}=\frac{2 e \sqrt{2 K K^{\prime}}}{Q^{2}}
$$

The amplitudes $B_{h_{e}}^{\lambda}$ for the diagrams in which the photon is emitted from the electron line are

$$
\begin{aligned}
B_{-}^{R}= & K^{\prime}\left(e^{i \phi} s_{\alpha^{\prime}} s+c_{\alpha^{\prime}} c\right)\left(e^{-i \phi} s_{\alpha^{\prime}} c-c_{\alpha^{\prime}} s\right) / 2 k^{\prime} \cdot q^{\prime} \\
& \times\left(P c_{\alpha^{\prime}} c_{\alpha}+Q^{\prime}\left(e^{i \phi} s_{\alpha^{\prime}} s+c_{\alpha^{\prime}} c\right)\left(e^{-i \phi} s_{\alpha} s+c_{\alpha} c\right)\right) \\
+ & \left(e^{-i \phi} s_{\alpha} c-c_{\alpha} s\right) /\left(-2 k \cdot q^{\prime}\right) \times\left[P c_{\alpha^{\prime}}\left(K c_{\alpha}\left(e^{i \phi} s_{\alpha} s+c_{\alpha} c\right)-Q^{\prime} c\right)\right. \\
& \left.+Q^{\prime}\left(e^{i \phi} s_{\alpha^{\prime}} s+c_{\alpha^{\prime}} c\right)\left(K\left(e^{i \phi} s_{\alpha} s+c_{\alpha} c\right)\left(e^{-i \phi} s_{\alpha} s+c_{\alpha} c\right)-Q^{\prime}\right)\right] \\
B_{+}^{R}= & K\left(e^{i \phi} s_{\alpha} s+c_{\alpha} c\right)\left(e^{-i \phi} s_{\alpha} c-c_{\alpha} s\right) /\left(-2 k \cdot q^{\prime}\right) \\
& \times\left(P c_{\alpha^{\prime}} c_{\alpha}+Q^{\prime}\left(e^{-i \phi} s_{\alpha^{\prime}} s+c_{\alpha^{\prime}} c\right)\left(e^{i \phi} s_{\alpha} s+c_{\alpha} c\right)\right) \\
+ & \left(e^{-i \phi} s_{\alpha^{\prime}} c-c_{\alpha^{\prime}} s\right) / 2 k^{\prime} \cdot q^{\prime} \times\left[P c_{\alpha}\left(K^{\prime} c_{\alpha^{\prime}}\left(e^{i \phi} s_{\alpha^{\prime}} s+c_{\alpha^{\prime}} c\right)+Q^{\prime} c\right)\right. \\
& \left.+Q^{\prime}\left(e^{i \phi} s_{\alpha} s+c_{\alpha} c\right)\left(K^{\prime}\left(e^{i \phi} s_{\alpha^{\prime}} s+c_{\alpha^{\prime}} c\right)\left(e^{-i \phi} s_{\alpha^{\prime}} s+c_{\alpha^{\prime}} c\right)+Q^{\prime}\right)\right]
\end{aligned}
$$

times the common factor

$$
c_{B}=\frac{2^{4} e^{3} \sqrt{2 K K^{\prime}}}{t_{p p^{\prime}}} F_{\pi}\left(t_{p p^{\prime}}\right)
$$


where $t_{p p^{\prime}}=-2 p \cdot p^{\prime}$ and $F_{\pi}$ is the pion form factor. The full amplitude is simply given by $A_{h_{e}}^{\lambda}+B_{h_{e}}^{\lambda}$. Combining our results and the data for the pion electromagnetic form factor a prediction for the high energy reaction $e \pi \rightarrow e \pi \gamma$ can be obtained.

We have tried three different forms for the pion wave-function denoted CZ [14], P2 and P3 [15]:

$$
\begin{aligned}
\phi_{C Z}(x) & =30 f_{\pi} x(1-x)(2 x-1)^{2} \\
\phi_{P 2}(x) & =6 f_{\pi} x(1-x)\left[-0.1821+5.91(2 x-1)^{2}\right] \\
\phi_{P 3}(x) & =6 f_{\pi} x(1-x)\left[0.6016-4.659(2 x-1)^{2}+15.52(2 x-1)^{4}\right]
\end{aligned}
$$

where $f_{\pi}=.133 \mathrm{GeV}$. For the strong coupling constant we have adopted the traditional value $\alpha_{s}=.3$. The expressions (27) for the wave function have all been derived with the sum rule method 16, 17.

The amplitudes for $\pi \gamma_{R} \rightarrow \pi \gamma_{R}(R R), \pi \gamma_{L} \rightarrow \pi \gamma_{R}(L R)$ and $\pi \gamma_{+} \rightarrow \pi \gamma_{R}(+R)$ are given in fig. 1 through 3 for different values of the photon virtuality $\eta=Q^{2} / 4 Q^{\prime 2}$. All other amplitudes can be obtained through parity transformations. The corresponding phases are given in fig. 4 through 6 .

The amplitudes show a complex dependence on the scattering angle and from the photon virtuality. The $+R$ amplitude grows as expected with $\eta$ and only for $\eta>.5$ gives a significant contribution. It peaks in the forward and backward direction and otherwise has a smooth behaviour with a phase close to $180^{\circ}$. The $L R$ amplitude is large in the backward direction and small for small scattering angles as a consequence of angular momentum conservation. It has a complicated dependence on $\eta$, decreasing for a while for increasing values of $\eta$ and then reversing the trend. The $R R$ amplitude is the only one with a non trivial phase for a real incoming photon and gives the largest contribution. It monotonically increases from small to large scattering angle for real photons. For virtual photons it presents a minimum at $\cos \theta \simeq-0.3$, which is quite deep for the $\mathrm{CZ}$ wave-function, and becomes much larger than the amplitude for real photons in the forward direction. Fig.4 shows that the minimum corresponds to a zero of the real part of the amplitude. The $R R$ contribution decreases rather smoothly with increasing $\eta$.

Our results for the $L R$ amplitude and for the real part of the $R R$ amplitude agree with the results of [9], which neglects all imaginary contributions.

The predictions do not depend too strongly on the choice of the wavefunction within the limited set which has been considered. With the exception of the $R R$ amplitude for the $\mathrm{CZ}$ wave-function and large virtualities, the amplitudes squared change at most by a factor of two or three for different wave-functions, in agreement with past experiences. The phases are also only moderately dependent upon the wave-function.

\section{Conclusions}

We have computed pion Compton scattering in perturbative QCD for real and spacelike initial photons.

We have studied three different methods for the convolution of the hard amplitude with the pion wave-functions. All procedures give the same results when properly 
applied. We find that a careful extrapolation to $\epsilon=0$ is needed in the $i \epsilon$ method. This may partially explain some disagreement between calculations performed in the past.

\section{Acknowledgements}

We wish to express our gratitude to G.R. Farrar and H. Zhang who independently computed the diagrams of the Compton set. We have checked that the two results are in complete agreement.

\section{References}

[1] J. Botts and G. Sterman, Phys. Lett. B224 (1989) 201, Erratum, ibid. B227 (1989) 501; Nucl. Phys. B325 (1989) 62.

[2] G. R. Farrar, G. Sterman and H. Zhang, Phys. Rev. Lett. 52 (1989) 2229.

[3] H.N. Li and G. Sterman, Nucl. Phys. B381 129 (1992)

[4] C. Corianò, A. Radyushkin and G. Sterman, Stony Brook Preprint ITP-SB-92-70, CEBAF Preprint CEBAF-TH-92-32.

[5] C. Corianò and Hsiang-nan Li, Academia Sinica Preprint IP-ASTP-18-92.

[6] E. Maina and G. Farrar, Phys. Lett. 206B (1988) 120.

[7] B. Nižić, Phys. Rev. D35 80 (1987). A.S. Kronfeld and B. Nižić, Phys.Rev. D44 (1991) 3445.

[8] G.R. Farrar and H. Zhang, Phys. Rev. D41. (1990) 3348.

[9] M. Tamazouzt, Phys. Lett. B211 (1988) 477.

[10] G. Berlad, A. Das, G. Eilam and J. Franklin, Ann. Phys. 75 (1973) 461.

[11] G. R. Farrar, private communication.

[12] G. Farrar and F. Neri, Phys. Lett. 130B (1983) 109.

[13] L. Wang, Phys. Rev. 142 (1966) 1187.

[14] V.L. Chernyak I.R. Zhitnitsky, Nucl. Phys. B201 (1982) 492.

[15] G. R. Farrar, K. Huleihel and H. Zhang, Nucl. Phys. B349 (1991) 655.

[16] M.A. Shifman, A.I. Vainshtein and V.I. Zakharov, Nucl. Phys. B147 (1979) 385, 448.

[17] V. L. Chernyak and A. R. Zhitnitsky, Phys. Rep. 112 (1984) 173 and references therein. 


\section{Figure Captions}

fig.1. $s^{3} \frac{d \sigma}{d \cos \theta}$ for $\pi \gamma_{R} \rightarrow \pi \gamma_{R}$ in the $\pi \gamma$ center-of-mass for the three different wavefunctions (a:CZ, b:P2, c:P3) and for different values of the photon virtuality $\eta=Q^{2} / 4 Q^{\prime 2}$.

fig.2. Same as in fig.1 for $\pi \gamma_{L} \rightarrow \pi \gamma_{R}$.

fig.3. Same as in fig.1 for $\pi \gamma_{+} \rightarrow \pi \gamma_{R}$.

fig.4. Phase in degrees for $\pi \gamma_{R} \rightarrow \pi \gamma_{R}$ as a function of the scattering angle in the $\pi \gamma$ center-of-mass for the three different wave-functions (a:CZ, b:P2, c:P3) and for different values of the photon virtuality.

fig.5. Same as in fig.4 for $\pi \gamma_{L} \rightarrow \pi \gamma_{R}$.

fig.6. Same as in fig.4 for $\pi \gamma_{+} \rightarrow \pi \gamma_{R}$. 